\title{
A standardized novel method to measure radiographic root changes following endodontic therapy in immature teeth
}

\author{
Natasha M. Flake, DDS, $\mathrm{PhD}^{1}$, Jennifer L. Gibbs, MAS, DDS, $\mathrm{PhD}^{1,2}$, Anibal Diogenes, MS, \\ DDS, PhD, Kenneth M. Hargreaves, DDS, PhD, and Asma A. Khan, BDS, PhD
}

\begin{abstract}
Introduction-Outcome studies of endodontic treatment of necrotic immature permanent teeth rely on radiographic measures as surrogates of whether the treatment achieved regeneration/ revascularization/revitalization. An increase in radiographic root length and/or width is thought to result in a better long-term prognosis for the tooth. In this study a method to measure radiographic outcomes of endodontic therapies on immature teeth was developed and validated.
\end{abstract}

\begin{abstract}
Methods-A standardized protocol was developed for measuring the entire area of the root of immature teeth. The "Radiographic Root Area" (RRA) measurement accounts for the entire surface area of the root as observed on a periapical radiograph. Reviewers were given instructions on how to measure RRA and they completed measurements on a set of standardized radiographs.
\end{abstract}

Results-The intra class correlation (ICC) between the four reviewers was 0.9945, suggesting high concordance among reviewers. There was no effect of reviewer on the measured RRA values. High concordance was also observed when one rater repeated the measurements, with an ICC value of 0.9995 . There was no significant difference in RRA values measured at the two sessions by the same rater. Furthermore, significant differences in RRA were detectable between clinical cases that demonstrated obvious continued root development and cases that did not demonstrate discernible root development.

Conclusions-These results suggest that RRA is a valid measure to assess radiographic outcomes in endodontically treated immature teeth, and RRA should be useful in future clinical studies of regenerative endodontic outcomes.

\section{Introduction}

Development of improved methods to treat immature necrotic teeth with open apices has been of considerable interest in endodontics in the past decade. Traditional treatment options include apexification with long term intracanal calcium hydroxide, or placement of a mineral trioxide aggregate (MTA) apical barrier prior to obturation of the root canal system (1). However, these treatment options result in a guarded long-term prognosis due to thin dentin walls, an incompletely formed apex, and in some cases short roots (2-5).

Furthermore, extraction is a poor alternative, because replacing the lost tooth with an

(C) 2013 American Association of Endodontics. Published by Elsevier Inc. All rights reserved.

${ }^{2}$ Corresponding Author: Jennifer L. Gibbs MAS, DDS, PhD, New York University College of Dentistry, Department of Endodontics, Clinic 7W, New York, NY 10010.

${ }_{1}$ Both authors contributed equally to the work.

Publisher's Disclaimer: This is a PDF file of an unedited manuscript that has been accepted for publication. As a service to our customers we are providing this early version of the manuscript. The manuscript will undergo copyediting, typesetting, and review of the resulting proof before it is published in its final citable form. Please note that during the production process errors may be discovered which could affect the content, and all legal disclaimers that apply to the journal pertain.

The authors deny any conflicts of interest. 
implant or fixed partial denture is contraindicated in young patients due to the mixed dentition status and ongoing cranio-skeletal development. Treatment methods variously termed "revascularization", "revitalization", or "regeneration" have become increasingly popular in endodontics. Although it is unknown if these procedures truly regenerate the pulp-dentin complex in patients, the term "regenerative endodontics" is often used to describe these procedures, is recognized by the American Association of Endodontists, and is widely used in the endodontic literature $(6,7)$. An advantage of regenerative endodontic treatment is further development of the root structure in the immature tooth (8). Increased thickness of the root dentin walls is believed to increase the long-term resistance to vertical root fracture for these teeth $(9,10)$. In addition, the regenerative endodontic procedure promotes apical closure, which will allow conventional non-surgical root canal therapy to be performed, should the tooth require endodontic therapy in the future.

To date, the published research on the outcomes of regenerative endodontic therapies includes a limited number of case series and retrospective studies $(3,11-14)$. These studies have all used radiographic measures as surrogates to assess the success of treatment on hard tissue deposition and to compare success of different treatments. Similarly, radiographic outcomes are routinely used in endodontic outcomes studies following both non-surgical and surgical endodontic therapy in mature teeth $(15,16)$. The rationale for using a radiographic outcome measure in regenerative endodontic studies is that an increase in radiographic root length and/or width is likely to result in greater resistance to root fracture (a common cause of eventual loss of teeth treated with calcium hydroxide apexification or an MTA apical barrier). It should be noted that, although it is assumed that the increased root length or width is due to pulpal regeneration and new dentin deposition, the nature of the tissue formed in the tooth cannot be known with certainty without adequate histological analysis.

Bose and colleagues published a retrospective evaluation of radiographic outcomes of cases after regenerative endodontic or control treatments (11). Preoperative and postoperative radiographs were analyzed in the program NIH Image J, and the plug-in application TurboReg was used to mathematically correct for differences in angulation between the preoperative and postoperative images. The root length from the CEJ to the root apex and the dentin thickness at the apical one third of the root were measured. Using this method, differences in root length and width between treatments were detectable. Subsequent studies of regenerative endodontic outcomes have employed this same method of measuring root length and width $(3,13)$. Although this method was able to detect differences between groups, it does have limitations, as it measures dentin thickness at only one level of the root. Furthermore, it is unknown if root length or width is a more important measure to predict clinical outcome, and at which level of the root the width is the most important. Chen and colleagues also reported a radiographic assessment of outcomes; cases were qualitatively scored for healing of the periapical lesion, thickening of the canal walls, and significantly continued root development (12). The authors summarized five types of responses to regenerative endodontic treatment as observed radiographically in these teeth. In another retrospective study, Chueh and colleagues recorded the final apical shape of the teeth after treatment (14). However, qualitative scoring systems are unlikely to have the desired sensitivity for use in future studies to compare outcomes between treatments.

The purpose of this study was to develop and validate a method to measure radiographic outcomes of endodontic therapies on immature teeth. The "Radiographic Root Area" (RRA) measurement accounts for the entire surface area of the root as observed on a periapical radiograph. RRA offers advantages over previously used radiographic outcomes methods, and we suggest that it will be useful in future clinical studies of on regenerative endodontic outcomes. 


\section{Methods}

\section{Measurement of Radiographic Root Area (RRA)}

Multiple individual radiographs were used to measure the RRA employing the freeware Image J (version 1.47; National Institutes of Health, Bethesda, MD). In Image J, the Polygon tool was used to outline the total root area, bordered on the occlusal aspect by the mesial and distal cemento-enamel junction (CEJ) and peripherally by the periodontal ligament space (Figure 1A \& 1C). The measurement function yields a value that reflects the entire planar area of the root. To account for the space taken by the root canal system, the polygon tool was again used to outline the root canal space (Figure 1B \& 1D), and the measurement for the area was obtained. The RRA measurement was calculated as the difference between total root area and the root canal space in each radiograph.

\section{Reproducibility of Radiographic Root Area Measurements}

To measure inter-rater reliability, four investigators (NF, AK, JG, AD) independently calculated RRA on 18 radiographs that were pre-operative and follow up radiographs of immature permanent teeth treated endodontically. Three investigators followed written instructions on how to perform the RRA measurements, and one had experience performing the measure previously (AD). To measure intra-rater reliability, one investigator (NF) remeasured RRA on 18 radiographs more than a year after the initial measures were taken. Reproducibility was assessed by calculating Intraclass Correlation (ICC) values. This is a measurement of concordance, similar to Kappa values, but can be used for continuous variables such as area. ICC was calculated using an online calculator (see Statistical Analysis).

\section{Radiographic Root Area in Test Set of Radiographs}

To test the validity of RRA as an outcome we calculated the RRA in pre-operative and follow up radiographs in a series of cases that were selected based on whether the case clearly demonstrated continued root development in the follow up radiograph or not. Seven cases were selected in each group. All cases in the positive root growth group were treated by regenerative procedures. The control cases that were selected based on not demonstrating any discernible root growth on follow up were either treated by regenerative procedures ( 5 cases) or MTA apexification (2 cases). The pre-operative and follow up radiographs in each case were normalized to each other using the TurboReg plug-in tool (Lausanne, VD, Switzerland) with the Image J software program (Bethesda, MD), to minimize distortions caused by variability in the angulation when the radiographs were acquired (11). The percent change in RRA was then calculated for each case.

\section{Statistical Analysis}

Differences between groups were assessed by one way ANOVA (inter- and intra-rater reliability) and a Student's t-test (2 group comparison of percentage change in RRA) (GraphPad Prism 5.0 for Mac OSX, 2010; La Jolla, CA). Concordance was measured with ICC using an online calculator (Dept. of Obstetrics and Gynecology, The Chinese University of Hong Kong, http://department.obg.cuhk.edu.hk/researchsupport/statmenu.asp).

\section{Results}

\section{Feasibility of RRA Measurements}

To determine the feasibility of learning to do RRA measurements, three endodontists were given instructions and images of examples on how to measure RRA using Image $\mathbf{J}$ software, as well as a practice set of radiographs The investigators had prior experience with image 
analysis, but no previous experience calculating RRA. Investigators were given instructions and images demonstrating how to outline the root area and root canal area (Figure 1). All investigators were able to complete the RRA measurement. Inter and intra rater reliability was then assessed.

\section{Inter and Intra Rater Reliability}

To assess the reliability of RRA as a radiographic outcome measure, four reviewers independently measured RRA in a set of standardized radiographs. The intra class correlation was 0.9945 , which suggests a high concordance amongst the reviewers. Further, on assessment of the results by ANOVA, there was no effect of reviewer on the observed RRA value (ANOVA $(3,17) \mathrm{F}=1.68 \mathrm{p}>0.05)$. We also used ICC to assess the intra-rater reliability and determine if the RRA measurements were reproducible in the same reviewer. One rater measured a series of radiographs and then repeated the same measurements more than one year later. Based on one reviewer, the analysis suggests high concordance with an ICC value of 0.9995 and ANOVA suggesting there was no difference between the values measured at the two times (ANOVA $(1,23) \mathrm{F}=0.52 ; \mathrm{p}>0.05$ ). This also demonstrates that the ability to measure RRA can be learnt with minimal training.

\section{Determination of Validity of RRA Measure using Selected Test Cases}

After demonstrating that the measurement of RRA was feasible by novice evaluators and that the measurements were reproducible, we wanted to assess whether RRA was able to clearly demonstrate differences in radiographs of clinical cases that show obvious continued root development after treatment, versus cases that did not demonstrate any discernible root development. We selected seven cases of each type and compared the change in RRA in standardized images. Figure 2 demonstrates two typical cases that were selected for the analysis. The control case demonstrates tooth $\# 9$, which was traumatized and underwent an apexification procedure with MTA placed in the apical region (Fig 2 A \& B). The calculated change in RRA for this case was $-2.8 \%$, which suggests that no clinically meaningful change in root area occurred in this case. The second case is a case of a traumatized tooth \#8 that underwent a standard regenerative treatment procedure, with obvious root thickening and lengthening observed on the radiograph taken at the recall appointment (Fig $2 \mathrm{C} \& \mathrm{D}$ ). The calculated change in RRA for this case was an increase of $48.6 \%$, which is suggestive of a clinically meaningful increase in root area. An average of $31 \%$ increase in RRA was detected in the group of selected cases with obvious root development (Fig 3, Mean: 31.6, SEM: 6.9), while the selected group of control cases showed negligible change (Fig 3, Mean: -0.6, SEM: 2.2). A statistically significant difference was observed between the two groups (Fig 3, t-test, $\mathrm{p}<0.001$ ). This finding supports the validity of RRA as a measure of root changes in endodontically treated immature teeth.

\section{Discussion}

In the present study, a new method, termed RRA, was developed and validated to assess radiographic outcomes of endodontic therapies in immature teeth. RRA measures the radiographic planar area of the root walls, but does not assess the periapical status. RRA showed high concordance between raters, and there was no significant effect of rater on the measurement. Furthermore, the intra-rater reliability was also high when the same rater analyzed radiographs more than one year after the first measurements. These results suggest that RRA is a consistent measurement that can be easily taught and calibrated among raters for use in clinical studies. Historically, assessment of radiographic outcomes of endodontic treatment has been inconsistent (17-20), but the validity of radiographic measures can be improved when raters are calibrated and standardized criteria are used $(15,21)$. In our study, there was high inter- and intra-rater reliability when measuring RRA. Importantly, raters 
received only written instructions on how to measure RRA prior to analyzing the radiographs. Although there was no significant effect of rater detected in this study, it is still ideal to have a consistent rater or group of raters measure all radiographs included in a study in order to minimize any differences. Furthermore, RRA was able to detect differences between control cases without obvious root growth and cases that were selected due to obvious root lengthening and thickening, suggesting that RRA is a sensitive measure that can detect potentially clinically meaningful differences in radiographic outcomes.

RRA offers advantages over previous methods used to assess radiographic outcomes of regenerative endodontic therapy. It accounts for the entire visualized planar surface area of the tooth. Thus, it is more comprehensive than a single linear measurement such as length or width. It is unknown at this time if root length or width is a more important surrogate measure for clinical outcome, and RRA accounts for changes in both dimensions. In addition, use of RRA does not require measuring root width at one arbitrary point on the root, which may be subject to the interpretation of the rater, and difficult to standardize across several radiographs. In cases where it is desirable to blind the rater to the treatment performed, it is possible for the canal space to be pseudocolored prior to measurement of RRA, in order to mask any treatment within the canal (Fig 1). Finally, use of a single outcome measure for all root growth allows more straightforward analysis in clinical studies, and RRA offers a simplified approach that will allow comparison between studies.

Although it has several advantages, measurement of RRA does have limitations. First, RRA can be difficult to measure in multi-rooted teeth when roots overlap. Only single-rooted teeth were used in the present study. Although there have been published cases of regenerative endodontic treatment in multi-rooted teeth, the majority of cases reported to date are in single-rooted teeth (those most likely to be affected by trauma and developmental anomalies) $(3,11-14,22)$. Second, as with the radiographic analysis method employed by Bose and colleagues, RRA relies on the program Turbo Reg to standardize preoperative and postoperative images (11). In order to minimize the correction that this program must accomplish, future prospective studies could employ a custom radiographic jig when exposing radiographs. However, a changing dentition in young patients may preclude the jig from fitting at follow-up visits. Third, measuring RRA does not account for the presence of intracanal calcifications, which occur in some cases treated with regenerative endodontic protocols (12). The cause of these calcifications and their effect on treatment outcome are unknown at this time. Finally, in some regenerative endodontic cases, the apex closes but there is minimal increase in root length or width (12). In these cases, the increase in RRA is likely to be minimal; however, this outcome may be considered a clinical success if there is resolution of signs and symptoms of apical periodontitis. Further, teeth with an apical constriction are better suited for conventional endodontic therapy in case there is recurrence of apical periodontitis.

Alternatives to RRA are available for assessing outcomes of endodontic therapy in immature teeth. The limitations of RRA associated with measuring multi-rooted teeth and the effects of changes in angulation between preoperative and follow-up images could be mitigated by using cone beam computed tomography (CBCT) to image these teeth. CBCT would allow individual roots to be analyzed without overlap from other roots or anatomic structures. This technology would also allow preoperative and postoperative images to be aligned based on coronal anatomy of the tooth. However, use of CBCT to follow-up solely for the purposes of research does not adhere to the principle of "as low as reasonably achievable" (ALARA), particularly in young patients (23). Finally, although observation of an increase in RRA may provide important clinical information relevant to the long term prognosis of the tooth, it should be recognized that RRA is only a surrogate measure for pulpal regeneration/ 
revascularization. Much further study is needed to identify the biologic processes contributing to continued root development in these cases.

In summary, RRA is a straightforward, valid measurement to assess radiographic changes in root size after endodontic treatment in immature teeth. The measurement is highly reproducible both between and within raters and should be useful in future research on regenerative endodontic outcomes.

\section{Acknowledgments}

We would like to acknowledge the contribution of Dr. Lina Maria Cortez at New York University in completing some of the RRA calculations and Dr. Jose Affonso de Almeida at Piracicaba Dental School-University of Campinas, for his early discussions on this project.. This work was partially supported by grants from the National Institutes of Health K23DE019461 (JLG), and R34 DE20864 (KMH).

\section{References}

1. Torabinejad M, Abu-Tahun I. Management of teeth with necrotic pulps and open apices. Endodontic Topics. 2012; 23:105-130.

2. Andreasen JO, Borum MK, Jacobsen HL, Andreasen FM. Replantation of 400 avulsed permanent incisors. 1. Diagnosis of healing complications. Endod Dent Traumatol. 1995; 11(2):51-58. [PubMed: 7641619]

3. Jeeruphan T, Jantarat J, Yanpiset K, Suwannapan L, Khewsawai P, Hargreaves KM. Mahidol study 1: comparison of radiographic and survival outcomes of immature teeth treated with either regenerative endodontic or apexification methods: a retrospective study. J Endod. 2012; 38(10): 1330-1336. [PubMed: 22980172]

4. Cvek M. Prognosis of luxated non-vital maxillary incisors treated with calcium hydroxide and filled with gutta-percha. A retrospective clinical study. Endod Dent Traumatol. 1992; 8(2):45-55. [PubMed: 1521505]

5. Andreasen JO, Farik B, Munksgaard EC. Long-term calcium hydroxide as a root canal dressing may increase risk of root fracture. Dent Traumatol. 2002; 18(3):134-137. [PubMed: 12110105]

6. Glossary of Endodontic Terms. American Association of Endodontists. 2012. http:// www.nxtbook.com/nxtbooks/aae/endodonticglossary/index.php\#/0

7. Regenerative Endodontics. Endodontics Colleagues for Excellence. 2013

8. Hargreaves KM, Diogenes A, Teixeira FB. Treatment options: biological basis of regenerative endodontic procedures. J Endod. 2013; 39(3 Suppl):S30-S43. [PubMed: 23439043]

9. Mireku AS, Romberg E, Fouad AF, Arola D. Vertical fracture of root filled teeth restored with posts: the effects of patient age and dentine thickness. Int Endod J. 2010; 43(3):218-225. [PubMed: 20158533]

10. Wilcox LR, Roskelley C, Sutton T. The relationship of root canal enlargement to finger-spreader induced vertical root fracture. J Endod. 1997; 23(8):533-534. [PubMed: 9587326]

11. Bose R, Nummikoski P, Hargreaves K. A retrospective evaluation of radiographic outcomes in immature teeth with necrotic root canal systems treated with regenerative endodontic procedures. $\mathrm{J}$ Endod. 2009; 35(10):1343-1349. [PubMed: 19801227]

12. Chen MY, Chen KL, Chen CA, Tayebaty F, Rosenberg PA, Lin LM. Responses of immature permanent teeth with infected necrotic pulp tissue and apical periodontitis/abscess to revascularization procedures. Int Endod J. 2011; 45(3):294-305. [PubMed: 22077958]

13. Cehreli ZC, Isbitiren B, Sara S, Erbas G. Regenerative endodontic treatment (revascularization) of immature necrotic molars medicated with calcium hydroxide: a case series. J Endod. 2011; 37(9): 1327-1330. [PubMed: 21846559]

14. Chueh LH, Ho YC, Kuo TC, Lai WH, Chen YH, Chiang CP. Regenerative endodontic treatment for necrotic immature permanent teeth. J Endod. 2009; 35(2):160-164. [PubMed: 19166764]

15. Orstavik D, Kerekes K, Eriksen HM. The periapical index: a scoring system for radiographic assessment of apical periodontitis. Endod Dent Traumatol. 1986; 2(1):20-34. [PubMed: 3457698] 
16. Friedman, S. Treatment outcome: the potential for healing and retained function. In: Ingle, J.; Bakland, L.; Baumgartner, J., editors. Endodontics. 6 ed. 2007. p. 1162-1232.

17. Goldman M, Pearson AH, Darzenta N. Endodontic success--who's reading the radiograph? Oral Surg Oral Med Oral Pathol. 1972; 33(3):432-437. [PubMed: 4501172]

18. Reit C, Hollender L. Radiographic evaluation of endodontic therapy and the influence of observer variation. Scand J Dent Res. 1983; 91(3):205-212. [PubMed: 6348935]

19. Zakariasen KL, Scott DA, Jensen JR. Endodontic recall radiographs: how reliable is our interpretation of endodontic success or failure and what factors affect our reliability? Oral Surg Oral Med Oral Pathol. 1984; 57(3):343-347. [PubMed: 6584824]

20. Goldman M, Pearson AH, Darzenta N. Reliability of radiographic interpretations. Oral Surg Oral Med Oral Pathol. 1974; 38(2):287-293. [PubMed: 4528712]

21. Orstavik D. Reliability of the periapical index scoring system. Scand J Dent Res. 1988; 96(2):108111. [PubMed: 3162597]

22. Law AS. Outcomes of regenerative endodontic procedures. Dent Clin North Am. 2012; 56(3):627637. [PubMed: 22835542]

23. Use of cone-beam computed tomography in endodontics Joint Position Statement of the American Association of Endodontists and the American Academy of Oral and Maxillofacial Radiology. Oral Surg Oral Med Oral Pathol Oral Radiol Endod. 111(2):234-237. 

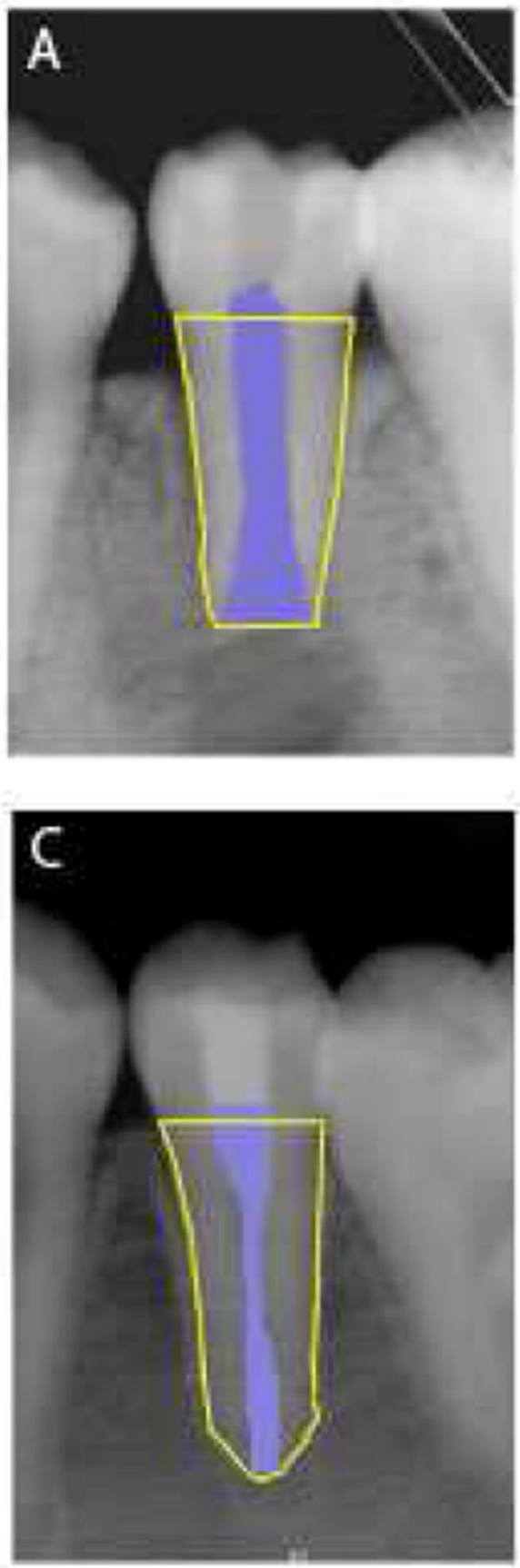

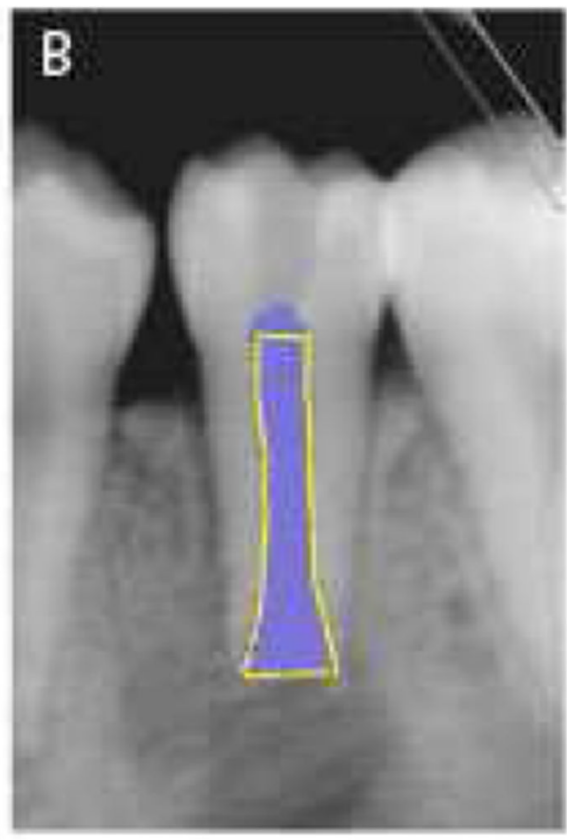

\section{D

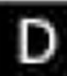

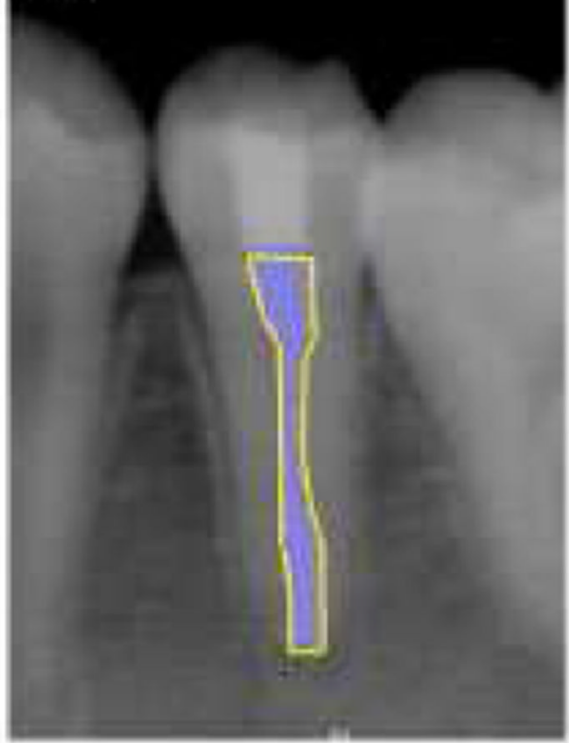

Figure 1.

Instructional images for performing radiographic root area measurements in Image J. For demonstration purposes the canal space of the images were masked with a purple color. This may be used to blind observers when needed. A: Masked preoperative radiograph demonstrating outlining of total root area. B: Masked preoperative radiograph demonstrating outlining of canal space. C: Masked follow-up radiograph demonstrating outlining of total root area. D: Masked follow-up radiograph demonstrating outlining of canal space. 


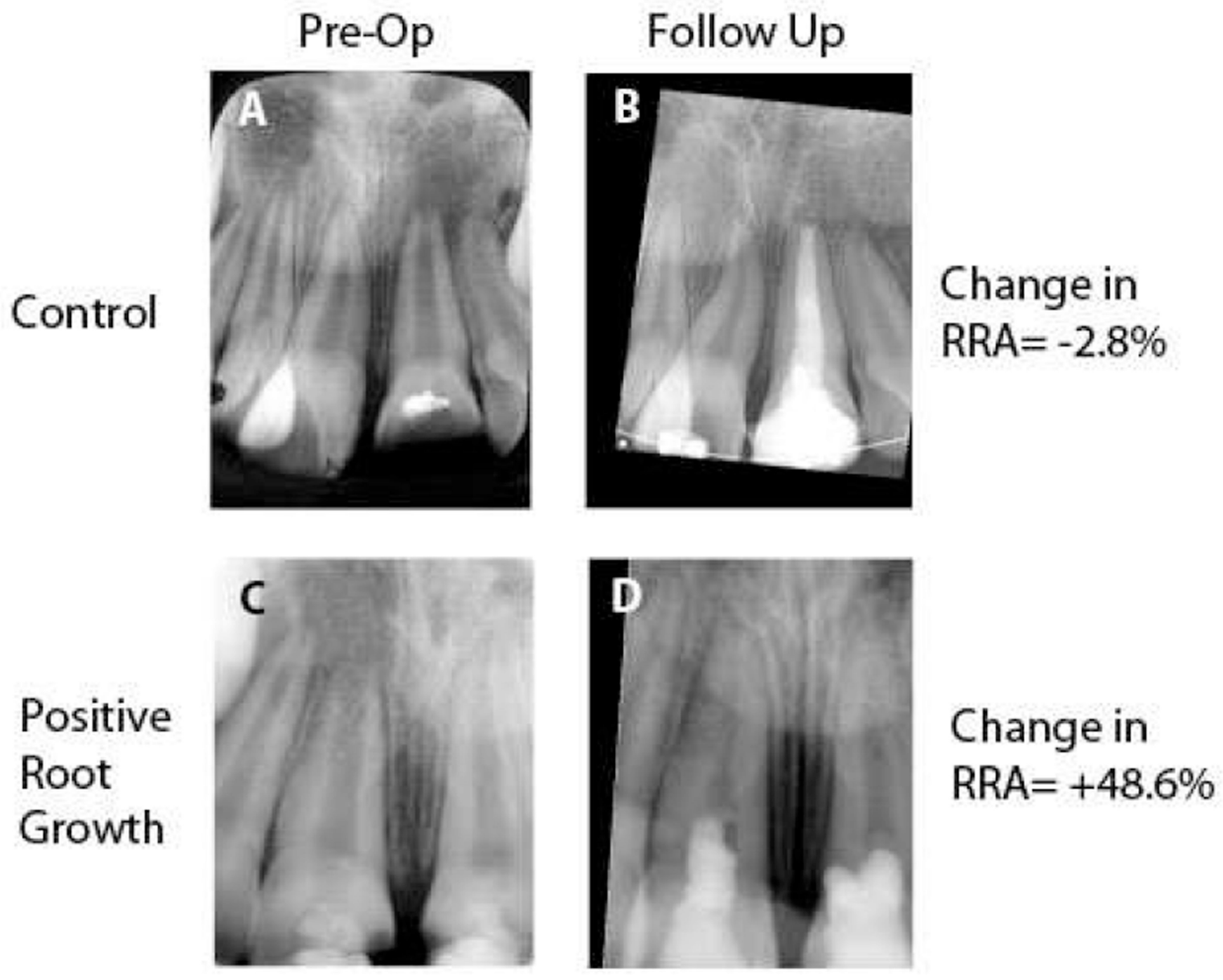

Figure 2.

Radiographs of representative cases used to test validity of RRA as an outcome to measure root development after endodontic treatment. A: Pre-operative radiograph of control case that was treated by MTA apexification B: Radiograph from follow up visit, demonstrating no obvious change in root area. Film was aligned and normalized to pre-operative film using the Turbo-Reg plug in, thus the image appears tilted, but the tooth in question is aligned between the two radiographs. The change in RRA calculated in this tooth was $-2.8 \%$. C: Pre-operative radiograph of a case selected that demonstrates obvious root growth after treatment. The tooth (\#8) was treated with a standard regenerative/revascularization protocol. B: Radiograph from follow up visit, demonstrating a clear increase in root width and length. Film was aligned and normalized to pre-operative film using the Turbo-Reg plugin. The change in RRA calculated for this tooth is $+48.6 \%$. Please note that tooth \#9 was not perforated, although the radiograph appears suggests a possible perforation. 


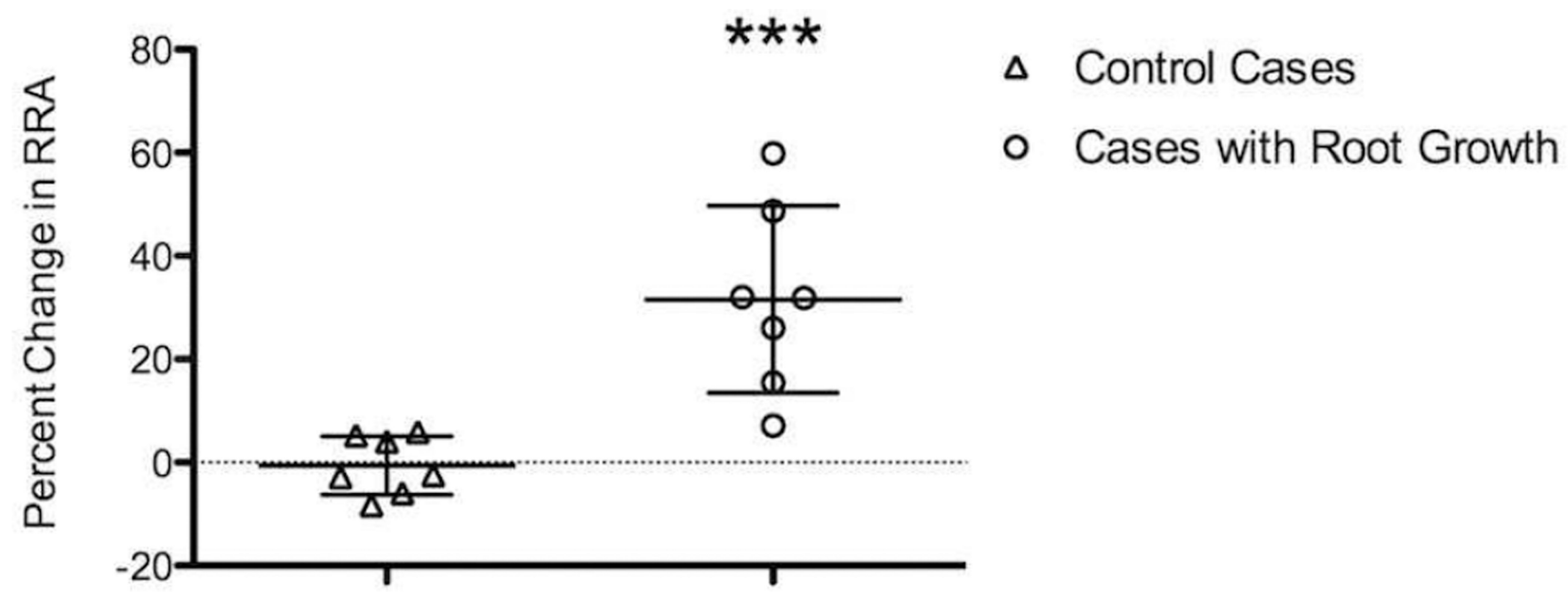

Figure 3.

Scatter plot of percentage change in RRA calculated from selected cases ( $n=7$ per group). An unpaired, 2-tailed t-test demonstrated a significant difference between groups $(\mathrm{p}=0.0008)$. Bars represent mean $\pm \mathrm{SD}$, open triangles represent measures from individual control cases, and open circles represent measures from individual cases demonstrating root growth. 